\title{
Comparison of subtalar mobilization with conventional physiotherapy treatment for the management of plantar fasciitis
}

\section{Muhammad Kashif ${ }^{1}$, Abdulaziz Aoudh Albalwi², Ahmed Abdullah Alharbi ${ }^{3}$,} Humaira Iram ${ }^{4}$, Nosheen Manzoor ${ }^{5}$

1,4,5 Department of Physical Therapy, Riphah College of Rehabilitation \& Allied Health Sciences, Riphah International University, Faisalabad, Pakistan.

2,3 Department of Physical Therapy, University of Tabuk, Tabuk, Saudi Arabia.

Correspondence: Muhammad Kashif. Email: kashif.shaffi@gmail.com

\section{$\underline{\text { Abstract }}$}

Objective: To compare the effectiveness of subtalar mobilisation technique on pain and functional disability compared to conventional physiotherapy in patients with planter fasciitis.

Method: The randomised controlled trial was conducted at the Prime Care Hospital, Faisalabad, Pakistan, from January to August 2017, and comprised patients of either gender aged 30-60 years presenting with complaints of heel and foot pain, a limited range of motion at the ankle joint due to heel pain, and pain in the morning when taking the first steps or after prolonged rest. The participants were randomly assigned to intervention group A, that received subtalar mobilisation, and control group B treated with therapeutic ultrasound. The groups received two treatment sessions per week over 3 weeks. Patients in both the groups received stretching and rigid tapping as standard treatment. Visual analogue scale and the foot and ankle disability inventory were used to measure pain and functional disability. Data was analysed using SPSS 20.

Results: Of the 60 patients enrolled, 8(13.3\%) were lost to follow-up, while 52(86.6\%) completed the study. Of the 52 subjects, there were $25(48 \%)$ in group A with a mean age of $32.40 \pm 8.02$, while in group B there were $27(52 \%)$ subjects with a mean age of $32.59 \pm 7.00$ years. Group A had 11(44\%) males and 14(56\%) females, while group B had 16(59.3\%) males and 11(40.7\%) females. Mean body mass index for group A was $25.35 \pm 3.8$ compared to $25.67 \pm 3.25$ for group B. There were significant differences in 
terms of pain between the two groups $(\mathrm{p}<0.05)$. Group A showed more reduction in

34 functional disability than group $B(p<0.05)$.

Conclusion: Subtalar mobilisation with movement was found to be effective in reducing pain and functional disability than conventional treatment in patients with 37 plantar fasciitis.

Key Words: Heel pain, Plantar fasciitis, Physiotherapy, Joint mobilisation, Taping.

\section{Introduction}

Plantar fasciitis (PF) is the most common cause of heel pain that affects $10-15 \%$ of the general population during their lifetime.(1) As non-inflammatory component is identified in recent literature in PF, the term may be modified as 'fasciosis' for a more appropriate description rather than 'fasciitis' that contains an inherent inflammatory component(2). PF is more prevalent than other foot pathologies that are presented in clinics, with an average of around two million people seeking treatment for it annually in the United States(3). A study conducted in Peshawar reported a prevalence of 13.2\% in the security population(4). Another study conducted among Pakistani teachers reported a $34.7 \% \mathrm{PF}$ prevalence(5). Overweight individuals, middle-aged women, athletes and male runners are mostly prone to plantar heel pain(6). There is an ongoing debate in literature about the causative factors of PF. Excessive loading of the plantar aponeurosis sometimes causes plantar fasciopathy(7).

Pain can present unilaterally or bilaterally during the initial steps in the morning or on resuming activity after an extended rest span. This distinctive presentation is often used to rule out other causative factors of heel pain(8). Cases with suspected fractures or with nerve entrapment in the tarsal tunnels can be excluded by the finding of increased pain symptomology on bearing weight on ankles. In adults, the bilateral pain around the ankles is referred to as Reiter's syndrome, from the family of reactive arthritis(9).

Various non-surgical treatments have been used to relieve the symptoms associated with heel pain. These include rest, active and passive range of motion (ROM), stretching and strengthening exercises, manipulation, mobilisation external support, including 
62 orthotics, night splints, and modalities such as therapeutic ultrasound with or without 63 the use of tropical application, electrical stimulation, whirlpool, and administration of non-steroidal anti-inflammatory drugs (NSAIDs) through iontophoresis $(6,10,11)$. Among these treatment methods, there is good evidence for the use of modalities and therapeutic exercises, but these are long-term approaches(12). Among conservative methods, the use of therapeutic ultrasound modality involves transmission of ultrasonic waves which increases tissue temperature and improves the extensibility of the fascia, thus stimulating the thermal cutaneous receptors. The non-thermal role of ultrasound is to modulate membrane properties, alter cell proliferation, and increase growth in proteins connected with inflammation and injury repair. Taken together, data suggests that non-thermal effects of therapeutic ultrasound can modify the inflammatory response(13). Thus, the inflammatory component of PF is managed by this non-thermal factor (14). A recent review of PF found that there is still a lack of high-quality evidence of any treatment modality (15).

Rigid taping is sometimes used for load reduction on the calcaneal attachment of the plantar fascia by supporting the longitudinal foot arches, minimising stress on the transverse arch, and limiting pronation in the forefoot(16). Taping manoeuvres shift the load from the medial longitudinal arch to the lateral border of the foot. This stress redirection limits the pronation in the foot, promoting the healing process(17). Increased use of taping has been found in literature for treating individuals with acute and subacute PF. The tape has also been used as a preventive measure for this condition(18). A comparative study among patients with PF to determine the efficacy of subtalar mobilisation techniques in combination with the conventional treatment protocols for any reduction in pain and disability level reported that benefit of subtalar mobilisation group was superior to conventional therapy alone in the management of patient with plantar heel pain, which indicated that the visual analogue scale (VAS) score between the two groups was significant. (19). Successful trials of conservative management of PF have been reported in literature $(20,21)$. There has been limited research about the use of manual therapy methods and their effectiveness in subjects diagnosed with 
$91 \mathrm{PF}(22)$. Therefore, the current study was planned to determine the effectiveness of

92 subtalar mobilisation versus conventional treatment on pain and functional disability in PF subjects.

\section{Patients and Methods}

The single-blind randomised controlled trial (RCT) was conducted at the Prime Care Hospital, Faisalabad, Pakistan, from January to August 2017. After approval from the ethics review committee of the local campus of Riphah International University, the sample size was calculated using $\mathrm{G}^{*}$ Power calculator(23) with 5\% margin of error, $80 \%$ power and effect size $d=0.74$ based on a previous study.(24). The sample was raised using purposive sampling technique from among patients of either gender aged 30-60 years presenting with complaints of heel and foot pain, limited ROM at the ankle joint due to heel pain, and pain in the morning when taking the first steps or after prolonged rest. Individuals with a history of trauma, acute or chronic pathology, any systemic illness, like rheumatism, a fracture below the knee during the preceding year, prior foot surgery, or a positive diagnosis of fat pad syndrome or tarsal tunnel syndrome were excluded.

After taking informed consent from the participants, they were randomised using computerised random numbers to intervention group A receiving subtalar mobilisation, and control group B receiving therapeutic ultrasound. The assessors were blinded to the treatment group to which a patient was allocated.

The intervention group A received subtalar mobilisation with movement through Mulligan technique for 15 minutes, stretching for 15 minutes and rigid tapping as standard treatment. Each patient received two treatment sessions per week for three weeks.

In the control group B, conventional physiotherapy included a session of therapeutic ultrasound for 15 minutes, stretching for 15 minutes and rigid tapping as standard treatment. Each patient received two treatment sessions per week for three weeks. 
Outcomes were measured using VAS and foot and ankle disability index (FADI)(25) at baseline, after 1st and 2nd week, and at the end of the treatment.

Data was analysed using SPSS 20. Quantitative variables were expressed as mean \pm standard deviation (SD). After application of analysis of variance (ANOVA), Tukey test was used for pairwise comparison for VAS and FADI within the groups. Independent samples t-test was used to find significant difference between the groups. $\mathrm{P} \leq 0.05$ was taken as statistically significant.

\section{Results}

Of the 60 patients enrolled, 8(13.3\%) were lost to follow-up, while 52(86.6\%) completed the study (Figure). Of the 52 subjects, there were 25(48\%) in group A with a mean age of $32.40 \pm 8.02$, while in group B there were $27(52 \%)$ subjects with a mean age of $32.59 \pm 7.00$ years. Group A had 11(44\%) males and 14(56\%) females, while group B had 16(59.3\%) males and 11(40.7\%) females. Mean body mass index (BMI) for group A was $25.35 \pm 3.8$ compared to $25.67 \pm 3.25$ for group B (Table 1).

There were significant differences in both the groups $(\mathrm{p}<0.05)$ except for VAS score at first week in groyp A (Table 2).

Both the groups showed improvement, but group A showed better outcome than group B (Table 3).

\section{Discussion}

In the current study, the experimental group had decreased signs and symptoms of PF, which helped in rapid healing. A similar study that compared the effects of taping techniques on reducing plantar fascia inflammation showed that subjects in the group in which taping was applied had faster decrease in their symptoms than did those in the other group $(\mathrm{p}=0.013)$. (26)

The current study used manual subtalar mobilisation with movement combined with taping techniques as the mainstay treatment for $\mathrm{PF}$ and compared this treatment with conservative treatment. The intervention manual treatment significantly reduced the 
mean pain profile, measured by VAS, on a sample of 30 participants. A study in 2015 (16) reported similar results, but that study had a sample size of just 30 participants divided into two groups. The control group also showed some improvement, but the difference was not significant. The finding is supported by other studies(20,21).

Only limited studies have been conducted in which large sample sizes were used to determine the effectiveness of manual mobilisation techniques. One case study included only four subjects who had complaints of inflammation of the plantar fascia. A combined treatment method of manual therapy (subtalar mobilisation joint glides) and stretching was applied for 8-10 months. The results of the treatment were compiled using assessment of pain reduction using a pain rating scale and a functional assessment. Mobilisation at the targeted joints resulted in reduction of pain and improvement in activities of daily living (27).

A study provided evidence that early subtalar mobilisation techniques are helpful in the reduction of complaints of pain in cases of plantar fascia inflammation(28). This supports the recommendations in recently published clinical practice guidelines for the treatment of PF which stated that, although weak, there is evidence that supports manual therapy as an effective treatment in the management of heel pain (29). A study (24) established that manual therapy is a better approach in treating patients with plantar heel pain. Decreased pain and improved foot function in people with PF are because subtalar mobilisation helps to increase the mobility of the calcaneus and talus, which decrease traction stress through the plantar fascia, which decreases pain and increases foot function(30).

The current study has limitations as the RCT was retrospectively registered with the World Health Organisation (WHO)-recognised Iranian Registry of Clinical Trials, with the registration number RCT20200221046567N2 on April 18, 2020.URL:https://www.irct.ir/trial/46988. Moreover, purposive sampling was used with limited generalisability and lack of control for confounding factors despite randomisation. Future studies on physiotherapy management of PF should investigate 
176 the effectiveness of different types of combination of mobilisation with movement

177 therapy with other electrotherapy modalities and should have long-term follow-up.

\section{Conclusion}

180 Individual with PF who received subtalar mobilisation with movement, stretching 181 exercise plus rigid taping showed significantly greater improvement in pain and 182 functional disability than conventional physiotherapy group which received ultrasound, 183 stretching exercise and rigid taping.

185 Disclaimer: None.

186 Conflict of interest: None.

187 Source of funding: None.

189 References

190 1. Thong-On S, Bovonsunthonchai S, Vachalathiti R, Intiravoranont W, Suwannarat

191 S, Smith R. Effects of strengthening and stretching exercises on the temporospatial gait 192 parameters in patients with plantar fasciitis: A randomized controlled trial. Ann Rehabil 193 Med. 2019;43(6):662.

194 2. Roxas M. Plantar fasciitis: diagnosis and therapeutic considerations. Altern Med 195 Rev 2005;10(2).

196 3. Pohl MB, Hamill J, Davis IS. Biomechanical and anatomic factors associated 197 with a history of plantar fasciitis in female runners. Clin J Sport Med 2009;19(5):3721986.

199 4. Abidin SZU, Haneef K, Malik NR, Mashal M, Zeb A, Rahman MU. 200 PREVELANCE AND ASSOCIATED RISK FACTORS FOR PLANTAR FASCIITIS 201 AMONG SECURITY FORCES PERSONNEL IN PESHAWAR. A of Allied Health 202 Sci 2019;5(2):20-3. 
203 5. Hashmi R, Naeem L, Arif S, Habiba U, Irfan R, Zafar M. Frequency of Plantar

204 Fasciitis among Females in Teaching Profession. J Aziz Fatimah Med \& Dent Coll. $205 \quad 2020 ; 2(2): 53-7$.

206 6. Hyland MR, Webber-Gaffney A, Cohen L, Lichtman SW. Randomized 207 controlled trial of calcaneal taping, sham taping, and plantar fascia stretching for the 208 short-term management of plantar heel pain. J Orthop Sports Phys Ther. 209 2006;36(6):364-71.

$2107 . \quad$ Rathleff M, Thorborg K. 'Load me up, Scotty': mechanotherapy for plantar 211 fasciopathy (formerly known as plantar fasciitis). BMJ Publishing Group Ltd and 212 British Association of Sport and Exercise Medicine; 2015.

213 8. Petraglia F, Ramazzina I, Costantino C. Plantar faseiitis in athletes: diagnostic 214 and treatment strategies. A systematic review. Ligaments Tendons J 2017;7(1):107-18.

2159 . Lee TG, Ahmad TS. Intralesional autologous blood injection compared to 216 corticosteroid injection for treatment of chronic plantar fasciitis. A prospective, 217 randomized, controlled trial. Foot Ankle Int. 2007;28(9):984-90.

218 10. Riddle DL, Pulisic M, Pidcoe P, Johnson RE. Risk factors for plantar fasciitis: a 219 matched case-control study. JBJS. 2003;85(5):872-7.

220 11. Fascia P. Diagnostic and Therapeutic Injection of the Ankle and Foot.

221 12. Pfeffer G, Bacchetti P, Deland J, Lewis A, Anderson R, Davis W, et al. 222 Comparison of custom and prefabricated orthoses in the initial treatment of proximal 223 plantar fasciitis. Foot \& Ankle Intl. 1999;20(4):214-21.

224 13. Johns LD. Nonthermal effects of therapeutic ultrasound: the frequency resonance 225 hypothesis. J Athl Train. 2002;37(3):293.

226 14. Gautham P, Nuhmani S, Kachanathu SJ. Plantar fasciitis: A review of literature. 227 Saudi J Med Med Sci 2014;14(2):69-73.

228 15. Dunning J, Butts R, Henry N, Mourad F, Brannon A, Rodriguez H, et al. 229 Electrical dry needling as an adjunct to exercise, manual therapy and ultrasound for 230 plantar fasciitis: A multi-center randomized clinical trial. PloS one. $231 \quad 2018 ; 13(10): \mathrm{e} 0205405$. 
232 16. Puttaswamaiah R, Chandran P. Degenerative plantar fasciitis: A review of current 233 concepts. The Foot. 2007;17(1):3-9.

234 17. Lange B, Chipchase L, Evans A. The effect of low-Dye taping on plantar 235 pressures, during gait, in subjects with navicular drop exceeding $10 \mathrm{~mm}$. J Orthop Sports 236 Phys Ther 2004;34(4):201-9.

237 18. Thomas JL, Christensen JC, Kravitz SR, Mendicino RW, Schuberth JM, Vanore $238 \mathrm{JV}$, et al. The diagnosis and treatment of heel pain: a clinical practice guideline-revision 239 2010. The Journal of Foot and Ankle Surgery. 2010;49(3):S1-S19.

240 19. Heggannavar A, Gupta RK. Effectiveness of Subtalar Joint Mobilization in 241 Plantar Heel Pain. Indian Journal of Physiotherapy and Occupational Therapy. $242 \quad 2015 ; 9(2)$.

243 20. Young CC, Rutherford DS, Niedfeldt MW. Treatment of plantar fasciitis. Am 244 Fam Physician. 2001;63(3):467.

245 21. Singh D, Angel J, Bentley G, Trevino SG. Fortnightly review: plantar fasciitis. 246 Bmj. 1997;315(7101):172-5.

247 22. Wang C-J, Wang F-S, Yang KD, Weng L-H, Ko J-Y. Long-term results of 248 extracorporeal shockwave treatment for plantar fasciitis. Am J Sports Med $249 \quad 2006 ; 34(4): 592-6$.

250 23. Faul F, Erdfelder E, Buchner A, Lang A-G. Statistical power analyses using G* 251 Power 3.1: Tests for correlation and regression analyses. Behav Res Methods. 252 2009;41(4):1149-60.

253 24. Cleland JA, Abbott JH, Kidd MO, Stockwell S, Cheney S, Gerrard DF, et al. 254 Manual physical therapy and exercise versus electrophysical agents and exercise in the 255 management of plantar heel pain: a multicenter randomized clinical trial. J Orthop 256 Sports Phys Ther. 2009;39(8):573-85.

257 25. Shazadeh Safavi P, Janney C, Jupiter D, Kunzler D, Bui R, Panchbhavi VK. A 258 systematic review of the outcome evaluation tools for the foot and ankle. Foot Ankle 259 Spec. 2019;12(5):461-70. 
26. Hyland MR, Webber-Gaffney A, Cohen L, Lichtman SW. Randomized

261

262

263

264

265

266

267

268

269

270

271

272

273

274

$275 \quad 2009 ; 30(4): 361-6$.

276

277

278

279

Table 1: Demographic data of the two groups.

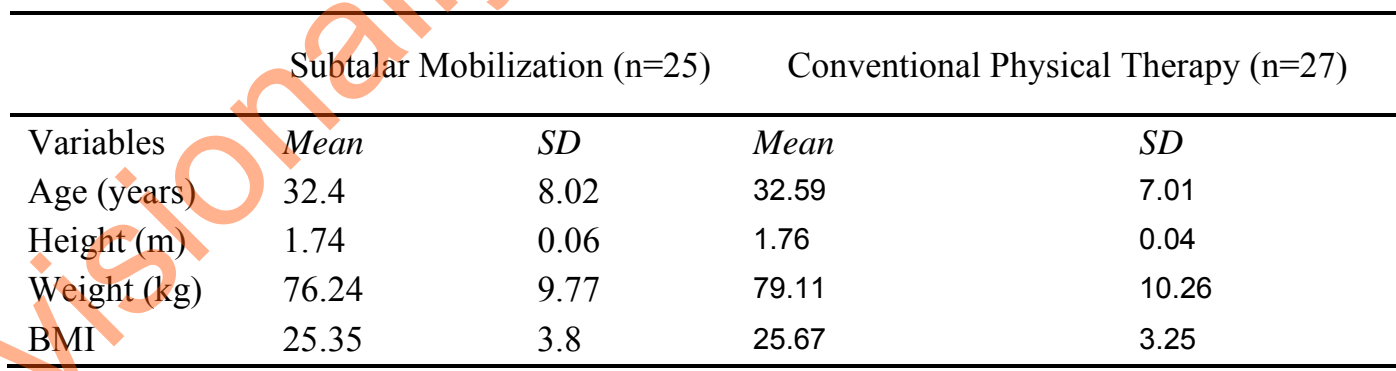

BMI: Body mass index. 
293

294

295

296

297

Table 2: Pairwise comparison of visual analogue scale (VAS) and foot and ankle disability index (FADI) scores within the groups.

\begin{tabular}{|c|c|c|c|c|c|c|}
\hline \multirow[t]{2}{*}{ Groups } & \multicolumn{3}{|c|}{ Subtalar Mobilization $(n=25)$} & \multicolumn{3}{|c|}{ Conventional Physical Therapy $(n=27)$} \\
\hline & Mean diff. & SE & P-value & Mean diff. & SE & P-value \\
\hline \multicolumn{7}{|l|}{ VAS } \\
\hline 0 vs 1 & 0.56 & 0.4 & 0.84 & $1.148^{*}$ & 0.276 & $<.001$ \\
\hline 0 vs 2 & $2.280^{*}$ & 0.248 & $<.001$ & $3.074^{*}$ & 0.272 & $<.001$ \\
\hline 0 vs 3 & $2.680^{*}$ & 0.355 & $<.001$ & $3.037^{*}$ & 0.28 & $<.001$ \\
\hline \multicolumn{7}{|l|}{ FADI } \\
\hline 1 vs 2 & $-12.04^{*}$ & 1.01 & $<.001$ & $-10.93^{*}$ & 1.214 & $<.001$ \\
\hline 1 vs 3 & $-22.76^{*}$ & 1.18 & $<.001$ & $-19.74^{*}$ & 1.304 & $<.001$ \\
\hline 1 vs 4 & $-36.92^{*}$ & 1.12 & $<.001$ & $-34.59^{*}$ & 1.466 & $<.001$ \\
\hline
\end{tabular}

SE: Standard error.

\section{Table 3: Intergroup data comparison.}

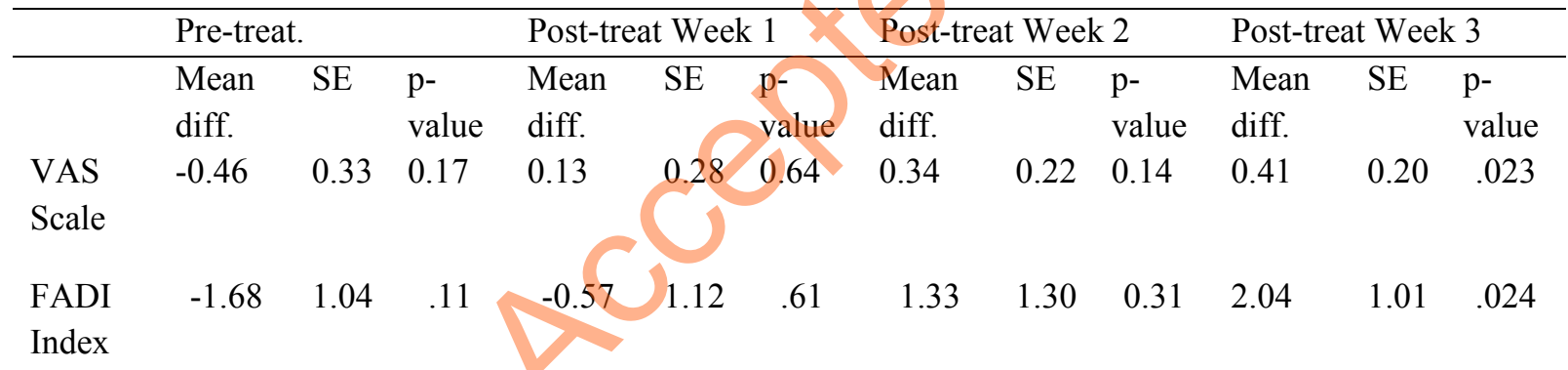

VAS: Visual analogue scale, FADI: Foot ankle disability index, SE: Standard error. 
308

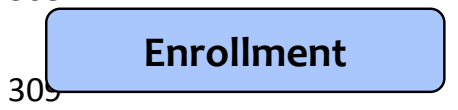

\section{Enrollment}

\section{Assessed for eligibility $(n=$}

71)

310

311

312

313

314
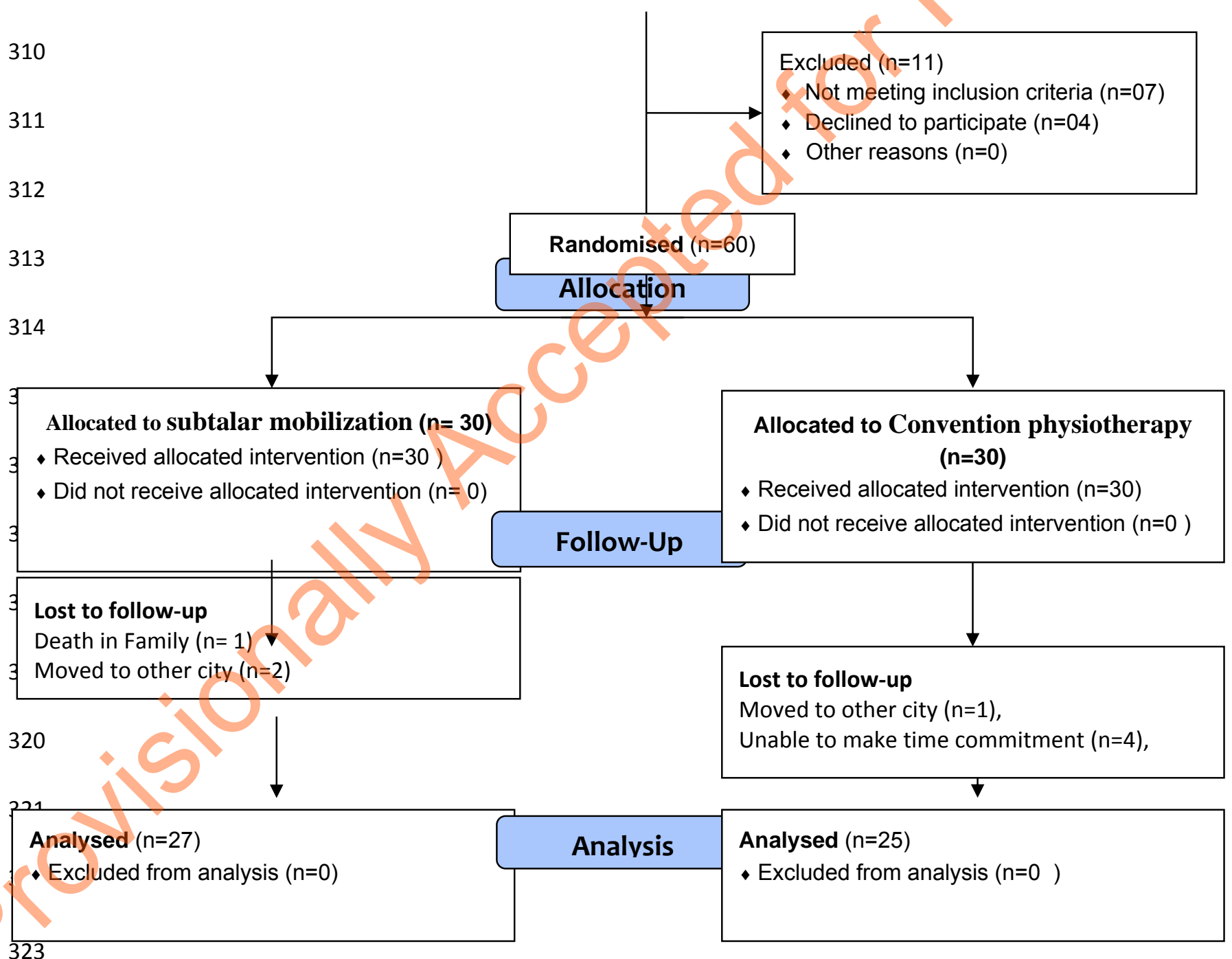

324 Figure: Study flow-chart. 